\title{
Caracterización epidemiológica y clínica de la infección por influenza A (H1N1) 2009 en San Felipe, Región de Valparaíso, Chile. Junio a agosto 2009
}

\author{
Maritza García, Elena Pedroni, Claudia González, Andrea Olea, Carmen Castillo, \\ Claudia Cameratti, Natalia Vergara y Rodrigo Fasce
}

Departamento de Epidemiología Ministerio de Salud Chile (MG, AO) Organización Panamericana de la Salud, Washington DC, E.U.A. (EP, CG) Servicio de Salud Aconcagua-Chile (CC, CC, NV) Instituto de Salud Pública-Chile (RF)

Conflictos de interés: no hay. Financiamiento: no hay.

Recibido: 4 de abril de 2012 Aceptado: 11 de junio de 2012

Correspondencia a: Maritza García maritzagarciaochoa@gmail.com

\section{Epidemiological and clinical characteristics of influenza A (H1N1) 2009 at San Felipe, Valparaiso Region, Chile. June-August 2009}

In order to understand the clinical and epidemiological aspects of infection with the AH1N1 influenza virus in Chile, a prospective study in the city of San Felipe was undertaken. It analyses the trends in consultations for respiratory causes in three primary care centers and hospital discharges by comparing data from years 2008 and 2009 until epidemiological week 37.It also includes a study of cases of ILI / SARI (influenza like disease/severe acute respiratory disease) in which viruses were detected by direct immunofluorescence (DIF) of nasopharyngeal aspirates and by real-time polymerase chain reaction in the case of influenza A (H1N1) 2009. A household survey was conducted in those cases with confirmed A (H1N1) infection, to identify contacts and history related to influenza virus transmission. The results indicate that the behavior of the pandemic was similar to that observed in the rest of the country, with an increase in emergency room visits for ILI. The most affected age group was from 5 to 14 years (26.5 per thousand inhabitants) and the least affected 60 years or older (1.2 per thousand). A 2.78\% of the cases corresponded to SARI and the fatality rate was $0.11 \%$.

Key words: Influenza AH1N1, AH1N1 Clinic, San Felipe AH1N1

Palabras claves: Influenza AH1N1, Clínica AH1N1, AH1N1 San Felipe.

\section{Introducción}

$\mathrm{E}$ n junio del año 2009, la Organización Mundial de la Salud (OMS) anunció una nueva pandemia debido a la emergencia y diseminación geográfica de un nuevo virus de Influenza tipo A (H1N1). El virus se detectó por primera vez en México y Estados Unidos de América produciendo infecciones respiratorias graves (IRAG) en un importante número de adultos jóvenes ${ }^{1}$. Los estudios virológicos realizados en el Centro de Control y Prevención de Enfermedades (CDC) de Atlanta y en la Agencia de Salud Pública de Canadá (PHAC) establecieron que el nuevo virus presentaba un triple reordenamiento de su material genético de virus de origen porcino, aviar y humano ${ }^{2}$.

Una vez declarada la emergencia, Chile activó el plan de preparación y respuesta para una pandemia de influenza desarrollado desde el año 2002, se fortaleció la vigilancia de virus respiratorios, detección y notificación de casos y brotes de enfermedad tipo influenza (ETI), se elaboraron guías clínicas para el diagnóstico y tratamiento de la infección, se reforzaron las medidas de control de infecciones asociadas a la atención de salud y se implementaron las medidas de salud pública tendientes a reducir el riesgo de transmisión de la infección en la comunidad ${ }^{3}$.
El 17 de mayo de 2009 se diagnosticaron los primeros casos de infección por virus influenza pandémico en la Región Metropolitana en adultos provenientes de República Dominicana y los primeros casos autóctonos en escolares ${ }^{3}$.

Al final de la primera ola pandémica el número de casos notificados alcanzó a 368.118 de los cuales 12.302 fueron confirmados por reacción de polimerasa en cadena en tiempo real (RPC-TR) $)^{3,4}$.

Como parte de la estrategia de vigilancia del virus pandémico, el Ministerio de Salud de Chile en colaboración con la Organización Panamericana de la Salud (OPS) y el CDC de Atlanta, E.U.A., realizó un estudio prospectivo para caracterizar epidemiológica y clínicamente la infección por el nuevo virus de influenza en la ciudad de San Felipe, provincia de San Felipe de la Región de Valparaíso. Se seleccionó esta comunidad dado que el inicio del brote de influenza fue más tardío que en la Región Metropolitana lo que permitió implementar el seguimiento.

San Felipe, es una ciudad localizada $70 \mathrm{~km}$ al norte de Santiago, con una población estimada para el año 2009 de 75.412 habitantes, cuenta con un hospital base tipo 2, un servicio de atención primaria de urgencia (SAPU), dos consultorios generales urbanos y un centro de atención primaria rural. 
Objetivo. Caracterizar aspectos epidemiológicos como curva epidémica, impacto de las consultas en los distintos niveles de atención, tasa de ataque y aspectos clínicos de la infección por el nuevo virus pandémico de influenza A (H1N1) 2009 en la comuna de San Felipe.

Descripción del estudio. Estudio de tendencia de consultas por IRA hasta la semana epidemiológica 37 durante los años 2008 y 2009.

Estudio descriptivo, prospectivo, de casos de IRAG confirmados por influenza A(H1N1) 2009 entre las semanas epidemiológicas 23 y 33.(23 de junio al 30 de agosto de 2009)

\section{Material y Método}

Estudio de tendencias. Se realizó una revisión de los registros de todas las consultas diarias del servicio de urgencia del Hospital San Camilo y de tres consultorios de atención primaria de la comuna, en el año 2008 y 2009 hasta la semana epidemiológica 37. Se registraron los siguientes diagnósticos: resfrío, resfrío común, virosis respiratoria, estado gripal, observación de influenza, influenza, gripe, infección respiratoria (IRA) alta, síndrome bronquial obstructivo, rinofaringitis, síndrome febril, neumonitis, neumonía y bronconeumonía.

Se revisaron los registros de todos los egresos con diagnóstico de neumonía, bronconeumonía y neumonitis del Hospital San Camilo entre las semanas 21 y la 28 de los años 2008 y 2009.

Estudio descriptivo prospectivo de casos de IRAG confirmados por influenza A (H1N1) 2009 semanas epidemiológicas 23 a 33. Se registraron en un formulario ad hoc los antecedentes epidemiológicos, demográficos y clínicos de todos los pacientes que consultaron con ETI y/o IRAG.

Se realizó cálculo de tasas de incidencia por grupos de edad utilizando para el denominador las estimaciones de población para la comuna elaboradas por el INE según el censo del año 2002.

Se aplicó encuesta durante visita domiciliaria para identificación y estudio de contactos de los casos de ETI y/o IRAG confirmados por RPC-TR.

\section{Población incluida en el estudio}

a) Todo residente de la comuna de San Felipe que cumplió con definición de caso de ETI: fiebre con temperatura axilar $>38^{\circ} \mathrm{C}$ y tos y al menos uno de los siguientes síntomas: mialgias, cefalea, odinofagia, rinorrea o congestión nasal y que consultó en la unidad de emergencia del Hospital San Camilo, SAPU o los centros de salud ambulatorios seleccionados. b) Todo residente de la comuna de San Felipe que cumplió con la definición de caso de IRAG: en los mayores de 5 años, presencia de dificultad respiratoria, taquipnea, cianosis o hipoxemia y en niños bajo 5 años de edad, deshidratación, rechazo alimentario o una consulta repetida por deterioro del cuadro clínico y que requirieron hospitalización en el Hospital San Camilo.

En todos los pacientes con ETI e IRAG se obtuvo una muestra de secreción respiratoria por aspirado nasofaríngeo para detección de virus respiratorios por inmunofluorescencia directa (IFD) e identificación de virus influenza A(H1N1) 2009 por RPC-TR. Las muestras fueron procesadas en el laboratorio clínico del Hospital San Camilo y confirmadas en el Instituto de Salud Pública (ISP).

A los pacientes con ETI o IRAG confirmados con RPCTR para influenza se les realizó una visita domiciliaria y se aplicó una encuesta epidemiológica para la identificación de contactos. Se registraron antecedentes epidemiológicos y clínicos relacionados con la transmisión de la influenza.

\section{Resultados}

Tendencia de consultas de urgencia y egresos hospitalarios 2008 y 2009. La comparación de las consultas diarias con diagnósticos de enfermedad respiratoria aguda, neumonía, neumonitis y bronconeumonía en el servicio de urgencia del hospital y consultorios de atención primaria de la comuna de San Felipe mostró que en el período comprendido entre las semanas 21 y 28 del 2008 se registraron 7.482 consultas (23,5\%); en cambio, en el año 2009 se registraron 11.738 consultas (40,8\%) (Tabla 1).

Durante el año 2009 se observó un aumento de consultas con el diagnóstico de influenza a partir de la semana epidemiológica 22 (31 de mayo al 6 de junio) hasta la semana 28 (12 al 18 de julio) comparado al 2008, donde el número de consultas aumentó en la semana 12 (16 al 22 de marzo) hasta la semana 21 (18 al 24 de mayo) (Figura 1).

En la Figura 2 se observa el aumento en el número de consultas por neumonía registradas entre las semanas 21 y 30 durante el año 2009 (483 consultas) comparado con el mismo período del año 2008 (140 consultas), esto es un aumento de $345 \%$.

Tabla 1. Consultas totales respiratorias en Servicio de

Urgencia, Hospital San Camilo y en Consultorios de

Atención Primaria, Comuna de San Felipe, 2008 y 2009

\begin{tabular}{|ccc|}
\hline $\begin{array}{c}\text { Semanas } \\
\text { epidemiológicas }\end{array}$ & $\mathbf{2 0 0 8}$ & $\mathbf{2 0 0 9}$ \\
\hline 1 a 37 & 31.847 & 28.787 \\
21 a 28 & $7.482(23,5 \%)$ & $11.738(40,8 \%)$ \\
\hline
\end{tabular}




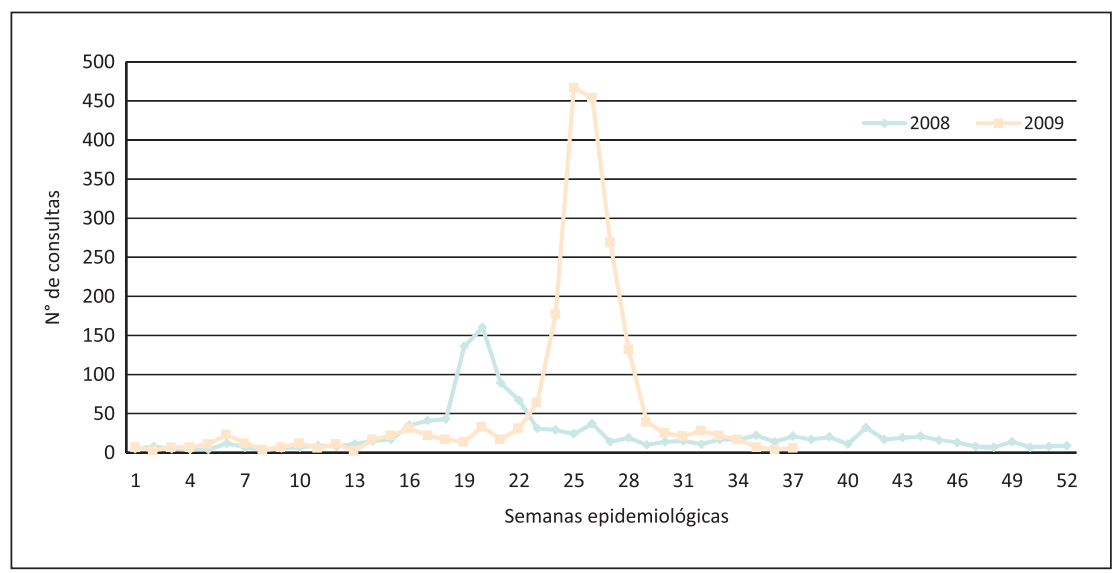

Figura 1. Consultas por influenza en Servicios de Urgencia y Atención Primaria de Salud, comuna de San Felipe, años 2008-2009.

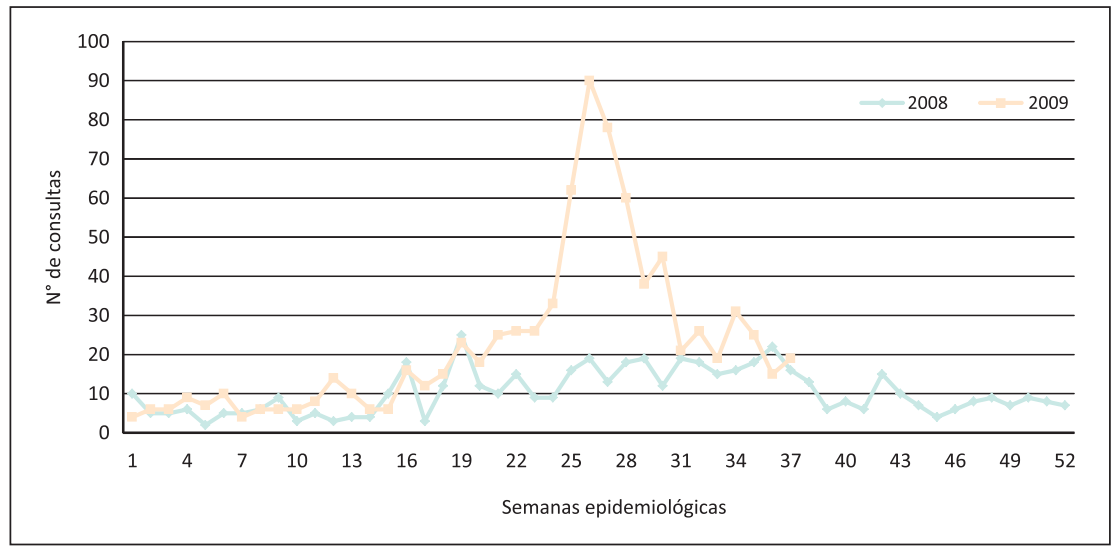

Figura 2. Consultas por neumonía en Servicios de Urgencia y Atención Primaria de Salud, comuna de San Felipe, años 2008-2009.
Caracterización de casos de enfermedad tipo influenza e infección respiratoria aguda grave. Durante el período estudiado (semanas epidemiológicas 23-33) se registraron 1.113 consultas por ETI e IRAG, 878 (78,9\%) fueron confirmados por RPC-TR. Se obtuvo datos completos en 791 pacientes, 22 (2,78\%) correspondieron a IRAG.

Características demográficas. El 54,6\% de los consultantes fueron mujeres. La mediana de edad fue 15 años, con un rango de 2 meses a 83 años. La mayor frecuencia de consultas se observó en el grupo etario entre 5 y 14 (37,6\%) seguido del grupo entre 15 y $29(31,3 \%)$ y la menor se observó en los mayores de $60(1,4 \%)$, seguido de los pacientes entre 45 y 59 (6,1\%) (Tabla 2).

La tasa más alta de incidencia se observó en el grupo de 5 a 14 años (26,5/1.000) seguida de los de 0 y 4 años $(15,4 / 1.000)$ y de 15 a 29 (15,2/1.000); en cambio, la menor tasa de incidencia se observó en los mayores de 60 años (1,2/1.000), seguido del grupo entre 45 y 59 años $(3,9 / 1000)$ (Figura 3).

Los síntomas más frecuentemente reportados en los casos de ETI fueron: fiebre (97,6\%), cefalea $(90,6 \%)$, mialgias (90,3\%), odinofagia (65,3\%), tos $(63,4 \%)$ y rinorrea (63,2\%). Otros síntomas y su duración se detallan en la Tabla 3.

Se pesquisaron 22 (2,78\%) casos de IRAG. La mediana de edad fue de 33 años con un rango de 2 meses a 83 años. Los síntomas más frecuentes reportados por este grupo fueron: tos (81,8\%), fiebre (77,3\%), disnea (50\%) y taquipnea (50\%) (Tabla 4). En 13 pacientes (59\%) se registró al menos una co-morbilidad (Tabla 5).

El diagnóstico de hospitalización más frecuente fue neumonía, 10 de 22 (45,5\%) (Tabla 6). De los 22 pacientes con IRAG, uno falleció durante el período en estudio y

Tabla 2. Distribución de casos confirmados influenza A (H1N1)2009, según edad y semana epidemiológica. San Felipe, Región de Valparaíso. Año 2009

\begin{tabular}{|c|c|c|c|c|c|c|c|}
\hline $\begin{array}{c}\text { Semana } \\
\text { epidemiológica }\end{array}$ & 0 a 4 años & 5 a 14 años & 15 a 29 años & 30 a 44 años & 45 a 59 años & 60 años y mas & Total \\
\hline 23 & 0 & 2 & 0 & 0 & 0 & 0 & 2 \\
\hline 24 & 1 & 1 & 0 & 1 & 0 & 0 & 3 \\
\hline 25 & 26 & 137 & 78 & 29 & 15 & 1 & 286 \\
\hline 26 & 34 & 115 & 116 & 50 & 15 & 4 & 334 \\
\hline 27 & 21 & 52 & 51 & 21 & 15 & 3 & 163 \\
\hline 29 & 0 & 1 & 3 & 1 & 1 & 1 & 7 \\
\hline 30 & 2 & 3 & 0 & 1 & 0 & 0 & 6 \\
\hline 31 & 0 & 0 & 1 & 1 & 1 & 0 & 3 \\
\hline 32 & 0 & 0 & 0 & 0 & 1 & 0 & 1 \\
\hline
\end{tabular}




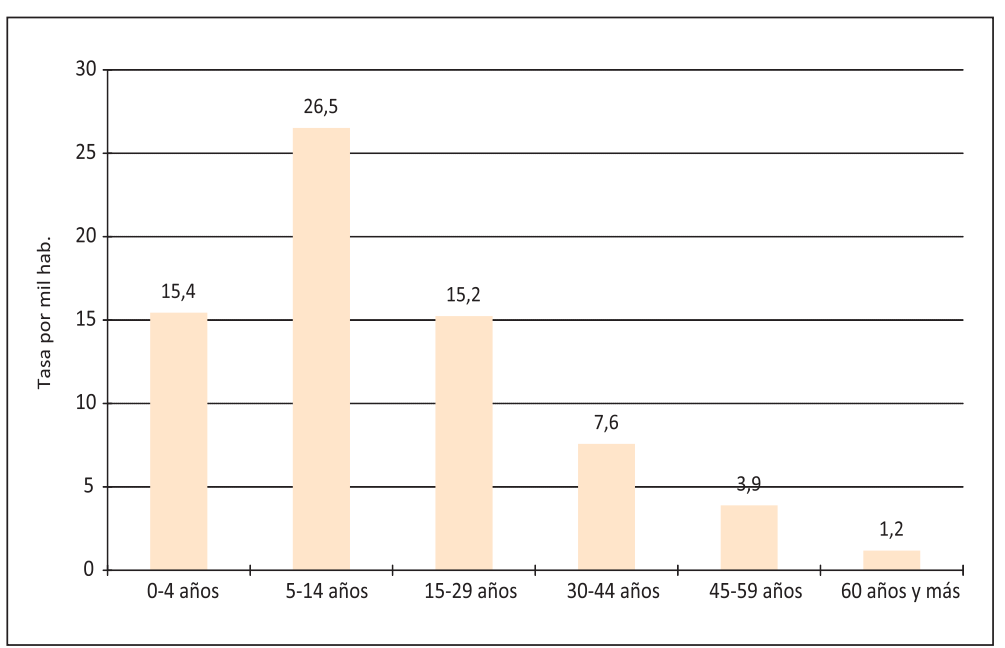

Figura 3. Tasa de incidencia de influenza A H1N1 por grupos de edad. Comuna de San Felipe, 2009 (tasa por mil habitantes).

Tabla 4. Frecuencia de síntomas y signos en casos infección respiratoria aguda grave. San Felipe, Región de Valparaíso. Año 2009

\begin{tabular}{|lcc|}
\hline Síntoma & N (22) & \% \\
\hline Tos & 18 & 81,8 \\
Fiebre $>=38^{\circ} \mathrm{C}$ & 17 & 77,3 \\
\hline Expectoración & 11 & 50,0 \\
\hline Disnea & 11 & 50,0 \\
\hline Taquipnea & 11 & 50,0 \\
\hline Cefalea & 9 & 41,0 \\
Vómitos & 6 & 27,3 \\
Mialgias & 6 & 27,3 \\
Odinofagia & 5 & 22,7 \\
Rinorrea & 5 & 22,7 \\
Náuseas & 4 & 18,2 \\
Artralgia & 3 & 13,6 \\
Diarrea & 1 & 4,5 \\
\hline
\end{tabular}

correspondió a un adulto de 78 años portador de diabetes mellitus, cardiopatía isquémica e insuficiencia renal crónica. La tasa de letalidad fue de 0,11\%.

Un 55,5\% de la muestras respiratorias resultaron (+)s por IFD para virus influenza A (H1N1) 2009, mientras que el porcentaje de positividad de las muestras respiratorias para influenza A (H1N1) 2009 por RPC-TR fue de $78,9 \%$

La Tabla 7 muestra los resultados de la IFD y RT-PCR para detección de virus influenza A (H1N1) 2009 en el período de estudio. El valor predictor (+) de la IF para detección de virus influenza A (H1N1) durante el brote pandémico fue de $97,2 \%$ y el valor predictor (-) de $44 \%$.

Se identificó co-infección en 32 pacientes (3,6\%) con otros virus respiratorios entre estos VRS, adenovirus y parainfluenza 3. El mayor número de co-infecciones se observó en el grupo de 5 a 14 años. La distribución por grupos de edad se presenta en la Tabla 8. No se detectó co- infección con los virus influenza B y parainfluenza 1 y 2.
Tabla 3. Distribución porcentual y días promedio de duración de síntomas en casos de enfermedad tipo influenza. San Felipe, Región de Valparaíso. Año 2009 (n: 1.113)

\begin{tabular}{|lcc|} 
Síntomas & $\%$ & Días promedio duración de síntomas \\
Fiebre $>=38^{\circ} \mathrm{C}$ & 97,6 & 2,47 \\
Cefalea & 90,6 & 3,36 \\
Mialgias & 90,3 & 3,66 \\
Odinofagia & 65,3 & 2,38 \\
\hline Tos & 63,4 & 3,57 \\
Rinorrea & 63,2 & 3,18 \\
Artralgia & 58,1 & 2,29 \\
Náuseas & 45,3 & 1,00 \\
Expectoración & 44,8 & 2,59 \\
Vómitos & 35,9 & 0,62 \\
Disnea & 30,7 & 1,04 \\
Diarrea & 26,6 & 0,61 \\
\hline
\end{tabular}

Tabla 5. Co-morbilidad en pacientes con infección respiratoria aguda grave. San Felipe, Región de Valparaíso. Año 2009

\begin{tabular}{|lc|}
\hline Co-morbilidad & $\mathbf{N}^{\mathbf{0}} \mathbf{1 3 / 2 2}$ \\
\hline Hipertensión arterial & 3 \\
\hline Insuficiencia renal crónica terminal & 2 \\
\hline Accidente vascular encefálico & 2 \\
\hline Síndrome de Timothy & 1 \\
\hline Hipertensión pulmonar & 1 \\
\hline Hipotiroidismo & 1 \\
\hline Diabetes mellitus tipo 2 & 1 \\
\hline Asma bronquial & 1 \\
\hline Obesidad & 1 \\
\hline Daño neurológico & 1 \\
\hline
\end{tabular}

Tabla 6. Diagnóstico de hospitalización de pacientes con infección respiratoria aguda grave. San Felipe, Región de Valparaíso. Año 2009

\begin{tabular}{|cc} 
Diagnóstico & $\mathbf{n}$ \\
Neumonía & 10 \\
Influenza & 2 \\
Síndrome febril & 2 \\
Asma bronquial & 2 \\
Taquiarritmia fetal & 1 \\
Sepsis & 1 \\
Bronconeumonía & 1 \\
SBOC & 1 \\
EPOC & 1 \\
Miopatía secundaria & 1 \\
Total & 22 \\
\hline
\end{tabular}

SBOC: síndrorme bronquial obstructivo crónico. EPOC: enfermedad pulmonar obstructica crónica. 


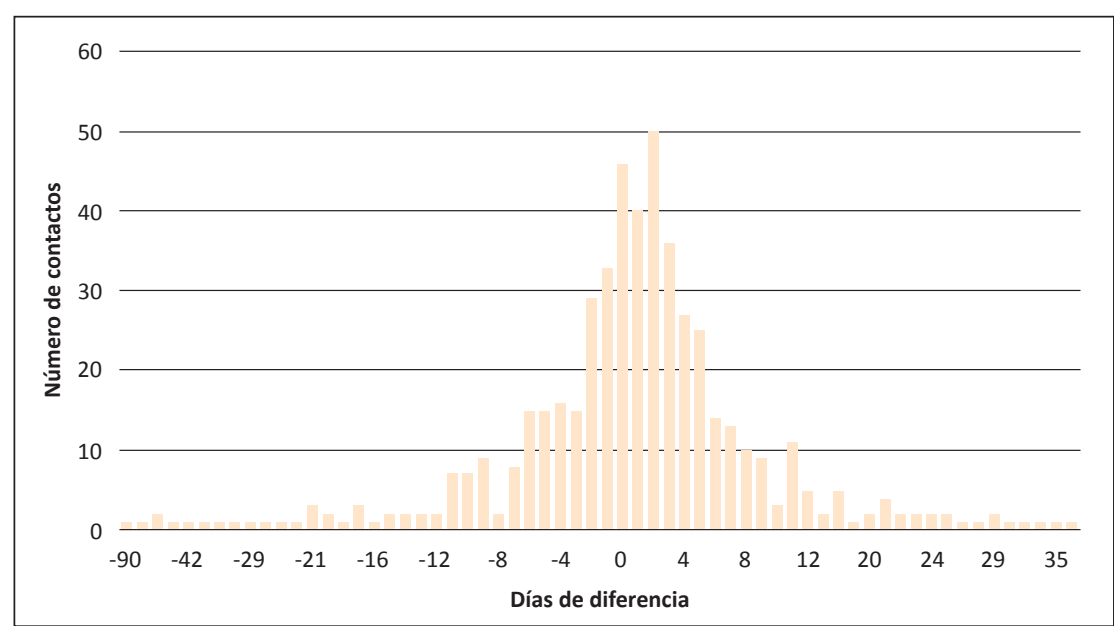

Figura 4. Diferencias en días entre la fecha de inicio de síntomas en los contactos y el inicio de síntomas en el respectivo caso confirmado ( $n=507$ contactos).

Tabla 8 . Co-infección de influenza detectada por IFD, en pacientes con enfermedad tipo influenza e infección respiratoria aguda grave, según grupos de edad y sexo. San Felipe, Región de Valparaíso. Año 2009

\begin{tabular}{|ccccccccc} 
& \multicolumn{2}{c}{ RPC-TR (+) } & \multicolumn{5}{c}{ IFD (+) } & IRAG \\
$\begin{array}{c}\text { Grupo de edad } \\
\text { (años) }\end{array}$ & Hombres & Mujeres & VRS & ADV & IA & P3 & Hospitalizados \\
\hline 0 a 4 & 43 & 48 & 8 & 0 & 60 & 0 & 4 \\
\hline 5 a 14 & 157 & 173 & 12 & 1 & 221 & 0 & 2 \\
\hline 15 a 29 & 118 & 157 & 1 & 3 & 200 & 0 & 4 \\
\hline 30 a 44 & 63 & 53 & 1 & 0 & 77 & 1 & 2 \\
\hline 45 a 59 & 21 & 33 & 2 & 0 & 37 & 0 & 7 \\
\hline 60 y + & 4 & 8 & 1 & 2 & 6 & 0 & 3 \\
\hline Total & $406(46,2 \%)$ & $472(53,8 \%)$ & 25 & 6 & 274 & 1 & 22 \\
\hline
\end{tabular}

VRS: virus respiratorio sincicial, ADV: adenovirus, IA: influenza A no tipificable. P3: Parainfluenza serotipo 3

Tabla 9. Distribución de contactos según sexo, edad y frecuencia de enfermedad tipo influenza. San Felipe, Región de Valparaíso. Año 2009

\section{$\mathbf{N}^{\circ}$ total de contactos}

\begin{tabular}{ccccccc}
$\begin{array}{c}\text { Grupo de edad } \\
\text { (años) }\end{array}$ & Hombres & Mujeres & Total & $\begin{array}{c}\text { Hombres } \\
(\%)\end{array}$ & $\begin{array}{c}\text { Mujeres } \\
(\%)\end{array}$ & $\begin{array}{c}\text { Total } \\
(\%)\end{array}$ \\
\hline 0 a 4 & 145 & 159 & 304 & $36(24,8)$ & $38(23,9)$ & $74(24,3)$ \\
\hline 5 a 14 & 248 & 278 & 526 & $72(29,0)$ & $90(32,4)$ & $162(30,8)$ \\
\hline 15 a 29 & 314 & 348 & 662 & $62(19,7)$ & $80(22,9)$ & $142(21,4)$ \\
\hline 30 a 44 & 291 & 382 & 673 & $45(15,4)$ & $55(14,4)$ & $100(14,8)$ \\
\hline 45 a 59 & 185 & 206 & 391 & $15(8,1)$ & $33(16,0)$ & $48(12,2)$ \\
\hline 6 o más & 69 & 101 & 170 & $3(4,3)$ & $9(8,9)$ & $12(7,0)$ \\
\hline Total & 1.252 & 1.474 & 2.726 & $233(18,6)$ & $305(20,7)$ & $538(19,7)$ \\
\hline
\end{tabular}

ETI: enfermedad tipo influenza.
Tabla 7. Valores predictores positivo y negativo de IFD para diagnóstico de Influenza A (H1N1) 2009. San Felipe, Región de Valparaíso. Año 2009

$\begin{array}{ll}\text { Sensibilidad } & 68,5 \% \\ \text { Especificidad } & 92,8 \% \\ \text { Valor Predictor Positivo } & 97,2 \% \\ \text { Valor Predictor Negativo } & 44,0 \%\end{array}$

Estudio de contactos. A 791 pacientes se les realizó encuesta y visita domiciliaria. Se pesquisaron 2.726 contactos (promedio de 3,4 contactos por caso). Un 19,7\% del total de contactos presentó síntomas de ETI. La mayor proporción de contactos que presentó síntomas correspondió a niños entre 5 y 14 años (30,8\%); en cambio, sólo 7\% de los contactos adultos mayores de 60 años presentaron síntomas (Tabla 9).

La encuesta epidemiológica identificó que algunos de los casos que ingresaron al estudio correspondían a casos secundarios y no al índice, ya que en el hogar había casos con inicio de síntomas de ETI antes que el caso que consultó (24\%). La Figura 4 muestra la distribución de contactos, según los días entre el inicio de síntomas del caso y el inicio de síntomas referido por sus contactos. El $39 \%$ de los contactos refirió inicio de síntomas entre las 0 y 48 horas desde la presentación del primer caso en el hogar.

\section{Discusión}

El 17 de mayo del 2009 se confirmaron en Chile los primeros dos casos de infección por virus influenza pandémico en turistas provenientes de República Dominicana. ${ }^{3}$

La detección de casos confirmados por virus pandémico en la ciudad de San Felipe fue más tardía que en otras zonas de nuestro país (semana epidemiológica 23).

Comparando iguales períodos entre el 2008 y 2009, se pudo confirmar que durante el año 2009 hubo un aumento importante tanto de consultas por ETI $(40,8 \%)$ y neumonía (56,8\%), que se detectaron a partir del 31 de mayo (semana epidemiológica 22).

Durante el año 2009, el mayor número de consultas por enfermedad respiratoria aguda, neumonía, neumonitis y bronconeumonía en el servicio de urgencia del hospital y consultorios de atención primaria de la comuna de San Felipe, se registró entre las semanas 21 y 28, coincidiendo con una alta circulación del virus pandémico. La comparación de las consultas diarias mostró que en el período comprendido entre las semanas 21 y 28 del 2008 se registraron 7.482 consultas (23,5\%); en cambio, el año 2009 se registraron 11.738 consultas (40,8\%).

$\mathrm{Al}$ igual que lo observado en el resto del país ${ }^{3}$ el grupo de edad más afectado fue entre 5 a 14 años (26,5/1000) y 
los menos afectados, los mayores de 60 años (1,2 / 1000).

Los síntomas más frecuentes de los casos de ETI fueron, fiebre $(97,6 \%)$, cefalea $(90,6 \%)$ y mialgias $(90,3 \%)$.

Del total de casos, 2,78\% requirió hospitalización, cifra mayor a la reportada a nivel nacional (0,46\%); sin embargo, la tasa de mortalidad observada fue menor a la registrada en el país $(0,11 \text { versus } 0,79 / 100.000)^{3,4}$. La causa más frecuente de hospitalización fue neumonía (45,5\%). Del total de casos de IRAG, 59\% tenía al menos una co-morbilidad y los factores de riesgo caracterizados fueron similares a lo descrito en otros estudios ${ }^{3,4}$.

El estudio de contactos permitió identificar 3,4 contactos por caso confirmado (19,7\%). Entre los contactos, el mayor número de sintomáticos se observó en el grupo de 5 a 14 años (30,8\%), y el menor fue el de los mayores de 60 años (7\%). El 39\% de los contactos inició síntomas respiratorios entre las 0 y 48 horas desde la presentación del primer caso en el hogar.

Sólo en 3,6\% de los casos se demostró co-infección viral y especialmente en el grupo de escolares.

\section{Conclusiones}

En la ciudad de San Felipe el comportamiento del virus pandémico fue similar a lo observado en el resto del país ${ }^{3}$. En este estudio se confirmó que las consultas por causa respiratoria aumentan principalmente en los servicios de atención de urgencia y son un indicador sensible para la vigilancia de eventos inusuales. Se evidenció que la confirmación del agente causal es más tardía ya que el estudio virológico no estaba ampliamente disponible. Dado que el aumento de las consultas por esta causa se produjo principalmente en los servicios de urgencia, la vigilancia epidemiológica, como estaba implementada hasta esa fecha, no logró una detección precoz ya que estaba concentrada en la detección de casos principalmente en los centros de atención primaria de salud. Este fenómeno también pudo detectarse en el brote de influenza pandémica (H1N1) en la Región de Los Lagos ${ }^{5}$ Entre las lecciones aprendidas durante la pandemia de influenza A (H1N1) 2009 destacan el mejoramiento de los sistemas de vigilancia, es decir la implementación del monitoreo del comportamiento de las consultas por ETI en servicios de urgencia como también las hospitalizaciones por IRAG y optimización de la capacidad diagnóstica y de laboratorio, entre otras.

\section{Resumen}

Con el objetivo de conocer los aspectos clínicos y epidemiológicos de la infección por el virus A (H1N1) en Chile, se realizó un estudio prospectivo en la ciudad de San Felipe, lugar donde el brote de influenza se inició tardíamente en relación a otras ciudades del país. Se analizó la tendencia en las consultas por causa respiratorias en tres centros de atención primaria y egresos hospitalarios comparando los años 2008 y 2009 hasta la semana epidemiológica 37, y se realizó un estudio prospectivo de casos de enfermedad tipo influenza/infección respiratoria aguda grave (ETI/IRAG), tomando muestras mediante aspirado nasofaríngeo para detección de virus respiratorios por inmunofluorescencia directa (IFD) e identificación de virus influenza A (H1N1) 2009 por reacción de polimerasa en cadena en tiempo real (RPC-TR). En los pacientes confirmados se hizo una encuesta en visita domiciliaria para identificación de contactos y antecedentes relacionados con la transmisión de la influenza. Los resultados indican que el comportamiento de la pandemia fue similar a lo observado en el resto del país, con aumento de consulta principalmente en los servicios de urgencia; el grupo de edad más afectado fue el de 5 a 14 años (26,5 por mil habitantes), el menos afectado el grupo mayor de 60 años (1,2 por mil habitantes). Un 2,78\% de los casos correspondió a IRAG y la tasa de letalidad fue de 0,11\%.

\section{Referencias bibliográfícas}

1.- CDC. Swine influenza A (H1N1) infection in two children. South California. MMWR Morb Mortal Wkly Rep 2009; 58: 400-2.

2.- Novel swine origin influenza A (H1N1) virus investigation team. Emergence of a novel swine origin influenza A (H1N1) virus in human. $\mathrm{N}$
Engl J Med 2009; 36.

3.- Influenza pandémica (H1N1) 2009. Ministerio de Salud de Chile. Reporte situación 2009. 13 de Enero 2010. Ultima vez visitado 21 de diciembre 2011. http://www.pandemia.cl/ templates/pandemia/documentos/Informe_13_ enero.pdf

4.- Dabanch J, Perret C, Najera M, González C, et al. Age as a risk factor for death from pandemic influenza (H1N1) 2009, Chile. Emerg Infect Dis 2011; 17 (7): 1256-8.

5.- Chilean task force for study of pandemic influenza A (H1N1). Outbreak of 2009 pandemic influenza A (H1N1), Los Lagos, Chile. April-June 2009. Eurosurveill 2010; 15 (1): pii19456. 